\title{
The effect of sediment content to fluid - solid interface time-domain waveform
}

\author{
Li Hua Qi ${ }^{1}$, Qing Bang Han ${ }^{1,2,{ }^{a},}$ Xue Ping Jiang ${ }^{1}$, Zi Wei Tong ${ }^{1}$, Yu Zhang ${ }^{1}$ and Chang Ping Zhu ${ }^{1}$ \\ ${ }^{1}$ College of IOT Engineering, Hohai University, Changzhou, Jiangsu, 213022, China \\ ${ }^{2}$ Huaian Research Institute, Hohai University, Huaian 223001, China
}

\begin{abstract}
A model was established for the fluid - solid interface of semi-infinite medium, and the characteristic equation was established using the potential function. Applying line source at the fluid-solid interface, transform solution of displacement could be obtained, and time-domain solution could be evaluated through the inverse Fourier transform. Two kinds of ultrasound suspension model, UA and HT, were used to study and discuss the effect of sediment content to fluid-solid interface time-domain waveform. The results of two models have a good consistency. The sediment content have a great impact on the Scholte wave. With the increase of the sediment content, for Scholte wave, the velocity increases and the attenuation decreases.
\end{abstract}

\section{Introduction}

The research on the effect of sediment content to fluid-solid interface time-domain waveform has profound theoretical and practical significance. In large projects, sediment content plays a key role on the implementation of the project. Since the ultrasonic wave penetrating effectively, there are obvious advantages in the detection of sediment contained of two-phase fluid ${ }^{[1]}$. In recent years, there are more and more application of ultrasonic testing. In this paper, we obtained the fluid-solid interface time-domain waveform by potential function. As early as 1948, Urick first proposed the form of velocity when the ultrasonic propagates in the suspension. Urick model is an ideal suspension model. Since then, a comprehensive model, Urick-Ament model, in which transmitted and reflected waves are considered. Then Harker and Temple proposed the Harker-Temple model based on the view of hydrodynamic. This paper mainly uses
Urick-Ament model (UA model) and coupling phase model (HT model) to study and discuss the effect of sediment content to fluid-solid interface time-domain waveform.

\section{Establishment of potential function}

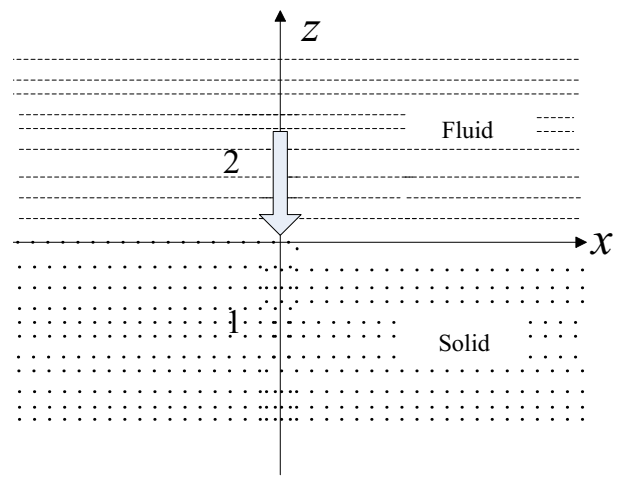

Figure1 Semi-infinite fluid-solid interface schematic

As shown in Figure 1, Z>0 for the semi-infinite fluid space, $\mathrm{Z}<0$ for the semi-infinite solid space, $\mathrm{Z}=0$ indicates the fluid - solid interface. The potential function of solid can be

\footnotetext{
a Corresponding author:hqb0092@163.com
} 
expressed as $\phi_{1} \quad \psi_{1}$ The potential function of fluid medium can be expressed as $\phi_{2}$. The displacement potential obeys the Helmholtz equation ${ }^{[2]}$.

$$
\left\{\begin{array}{l}
\nabla^{2} \phi=\frac{\partial^{2} \phi}{c_{L}^{2} \partial^{2} t^{2}} \\
\nabla^{2} \psi=\frac{\partial^{2} \psi}{c_{S}^{2} \partial^{2} t^{2}}
\end{array}\right.
$$

where $c_{L}$ is the velocity of longitudinal wave, $c_{S}$ is the velocity of shear wave. Carrying out Fourier transform to space $x$ and time $t$

$$
\begin{aligned}
& G(k)=\int_{-\infty}^{+\infty} g(x) e^{i k x} d x \\
& F(\omega)=\int_{-\infty}^{+\infty} f(t) e^{-i \omega t} d t
\end{aligned}
$$

For the semi-infinite solid medium

$$
\left\{\begin{array}{l}
\bar{\phi}^{*}=A_{1} e^{-k_{a 1} z} \\
{\overline{\psi_{1}}}^{*}=C_{1} e^{-k_{b 1} z}
\end{array}\right.
$$

where, $k_{a 1}=\sqrt{k^{2}-\frac{\omega^{2}}{c_{L 1}{ }^{2}}} \quad k_{b 1}=\sqrt{k^{2}-\frac{\omega^{2}}{c_{S 1}{ }^{2}}}$

Displacement, stress can be expressed by potential function as

$$
\left\{\begin{array}{l}
{\overline{\mu_{z 1}}}^{*}=-k_{a 1} e^{-k_{a 1} z} A_{1}+i k e^{-k_{b 1} z} C_{1} \\
{\overline{\sigma_{z z 1}}}^{*}=\mu_{1}\left(2 k^{2}-\frac{\omega^{2}}{c_{S 1}{ }^{2}}\right) e^{-k_{a 1} z} A_{1}-2 \mu_{1} i k k_{b 1} e^{-k_{b l} z} C_{1} \\
{\overline{\sigma_{z x 1}}}^{*}=-\mu_{1}\left(2 k^{2}-\frac{\omega^{2}}{c_{S 1}{ }^{2}}\right) e^{-k_{b \mid} z} C_{1}-2 i k k_{a 1} e^{-k_{a 1} z} A_{1}
\end{array}\right.
$$

For the semi-infinite fluid medium

$$
\bar{\phi}_{2}^{*}=B_{2} e^{k_{a 1} z}
$$

where, $k_{a 2}=\sqrt{k^{2}-\frac{\omega^{2}}{c_{L 2}^{2}}}$

Displacement, stress can be expressed by potential function as

$$
\left\{\begin{array}{l}
{\overline{\mu_{z 2}}}^{*}=k_{a 2} e^{k_{a z} z} B_{2} \\
{\overline{\sigma_{z z 2}}}^{*}=-\lambda_{2} \frac{\omega^{2}}{c_{L}^{2}} e^{k_{a 2} z} B_{2} \\
{\overline{\sigma_{z x 1}}}^{*}=0
\end{array}\right.
$$

At $\mathrm{z}=0$, According to the boundary conditions:

$$
\left\{\begin{array}{l}
{\overline{\mu_{z 1}}}^{*}={\overline{\mu_{z 2}}}^{*}{\overline{\sigma_{z z 1}}}^{*}-{\overline{\sigma_{z z 2}}}^{*}=G(k) F(w) \\
{\overline{\sigma_{z x 1}}}^{*}={\overline{\sigma_{z x 2}}}^{*}=0
\end{array}\right.
$$

we can get the characteristic equation:

$$
\left[\begin{array}{lll}
m_{11} & m_{12} & m_{13} \\
m_{21} & m_{22} & m_{23} \\
m_{31} & m_{32} & m_{33}
\end{array}\right]\left[\begin{array}{l}
A_{1} \\
B_{2} \\
C_{1}
\end{array}\right]=\left[\begin{array}{c}
0 \\
G(k) F(w) \\
0
\end{array}\right]
$$

$m 11=-k_{a 1} \quad m 12=k_{a 2} \quad m 13=i k$

$m 21=-\mu_{1}\left(2 k^{2}-\frac{\omega^{2}}{c_{S 1}^{2}}\right)$

$m 22=-\lambda_{2} \frac{\omega^{2}}{c_{L 2}^{2}} \quad m 23=2 i k \mu_{1} k_{b 1}$

$m 31=2 i k k_{a 1} \quad m 32=0 \quad m 33=2 k^{2}-\frac{\omega^{2}}{c_{S 1}{ }^{2}}$

By the formula (8), coefficients $A_{1} \quad B_{2} \quad C_{1}$ can be obtained, then the transform solution of displacement of solid can be obtained.

$$
{\overline{\mu_{z 1}}}^{*}=-k_{a 1} A_{1}+i k C_{1}
$$

Inverse transform yields the solution of displacement

$$
\mu_{z 1}=\int_{-\infty}^{+\infty}\left(\int_{-\infty}^{+\infty}{\overline{\mu_{z 1}}}^{*} e^{-i k x} d k\right) e^{i \omega t} d \omega
$$

Formula(8), $G(k) \quad F(\omega)$ are obtained from transformed $g(x) \quad f(t)$

$$
\begin{aligned}
& G(k)=\int_{-\infty}^{+\infty} g(x) e^{i k x} d x \\
& F(\omega)=\int_{-\infty}^{+\infty} f(t) e^{-i \omega t} d t
\end{aligned}
$$

This passage selects $G(k)=1 \quad F(\omega)=e^{\frac{-\left(f-f_{0}\right)^{2}}{b^{2}}}$

\section{Theoretical models of the suspension}

\subsection{Urick and Ament model}

It is a true model of fluid ${ }^{[3-4]}$, taking into account the refraction, reflection and other complex situations which occur during the propagation of the wave. The complex propagation equation of $k_{s}^{2}$, which describes both the velocity and attenuation in the suspension is:

$k_{s}^{2}=k^{2} \frac{\beta_{e f f}}{\beta_{c}} \times\left[1+\frac{3 \varphi \xi(b R(2 b R+3)+3 i(b R+1))}{b R(4 \xi b R+6 b R+9)+9 i(b R+1)}\right]$ 
Where $b=\left(\frac{\omega \rho}{2 \alpha}\right)^{1 / 2}, \xi=\frac{\rho^{\prime}-\rho}{\rho}, \alpha$ is viscosity of water, $R$ is the radius of suspended sediment particles, $k$ is real wave number of the pure liquid. $k_{s}$ is the plural wave number, whose real part represents real wave number, imaginary part denotes attenuation.

Substituting equation(12) into.(8), the time-domain waveforms of different sediment content can be obtained.

\subsection{Hanker-Temple model}

Harker and Temple ${ }^{[5-6]}$ considered suspension of wave phenomena from hydrodynamics, and deduced the viscous drag equation, momentum and mass conservation equations. The plural wave number equation of the Harker-Temple model could be obtained by solving these differential equations:

$k_{l}^{2}=\beta_{e f f} \omega^{2}\left[\frac{\rho\left\{\rho^{\prime}+\left(\varphi \rho^{\prime}+(1-\varphi) \rho\right) \mathrm{S}(\omega)\right\}}{\left(\varphi \rho+(1-\varphi) \rho^{\prime}\right)+\rho \mathrm{S}(\omega)}\right]$

Where $S(\omega)=\frac{1+2 \varphi}{2(1-\varphi)^{2}}+\frac{1}{1-\varphi} \frac{9 \varepsilon_{v}}{4}\left[1+\left(1+\frac{\varepsilon_{v}}{R}\right) \mathrm{i}\right], \rho$ is the density of continuous phase medium, $\rho^{\prime}$ is the density of particle phase, $\varphi$ is the solid volume fraction, $\beta_{\text {eff }}$ is effective density volume compressibility factor, $R$ is the radius of suspended sediment particles, $\varepsilon_{v}=\sqrt{2 \eta / \omega \rho}$ is viscous shin depth, $\eta$ is viscosity.

The velocity $c_{l 1}$ of the fluid can be obtained through the equation $k_{l}=\omega / c_{l 1}$. Then substituting $c_{l 1}$ in to $\mathrm{Eq}(5)$, the time-domain waveforms of different sediment content can be obtained.

\section{Numerical results}

Parameters of Semi-infinite medium and sediment are listed in Table 1 and Table 2.Numerical results are shown in Figure 2 to Figure 7.

Table 1. Material parameters

\begin{tabular}{|c|c|r|c|}
\hline \multirow{3}{*}{ Media } & $\begin{array}{c}\text { Longitudinal wave } \\
\text { velocity } /\left(\mathrm{m} \cdot \mathrm{s}^{-1}\right)\end{array}$ & $\begin{array}{r}\text { Shear wave } \\
\text { velocity } /\left(\mathrm{m} \cdot \mathrm{s}^{-1}\right)\end{array}$ & $\begin{array}{c}\text { Density } \\
(\mathrm{kg} \cdot \\
\left.\mathrm{m}^{-3}\right)\end{array}$ \\
\hline
\end{tabular}

\begin{tabular}{|c|c|c|c|}
\hline Aluminum & 6260 & 3080 & 2700 \\
\hline water & 1500 & -- & 1000 \\
\hline Media & $\begin{array}{l}\text { Density } \\
/\left(\mathrm{kg} \cdot \mathrm{m}^{-3}\right)\end{array}$ & $\begin{array}{l}\text { Longitudinal } \\
\text { wave } \\
\text { velocity } /\left(\mathrm{m} \cdot \mathrm{s}^{-1}\right)\end{array}$ & $\begin{array}{c}\text { Shear wave } \\
\text { velocity } /\left(\mathrm{m} \cdot \mathrm{s}^{-1}\right)\end{array}$ \\
\hline water & 1000 & 1500 & -- \\
\hline Sediment & 2640 & 6600 & 2750 \\
\hline
\end{tabular}

\begin{tabular}{|c|c|c|c|}
\hline Media & $\begin{array}{c}\text { volume } \\
\text { compressibi } \\
\text { lity } \\
/\left(10^{10} \mathrm{pa}\right)\end{array}$ & $\begin{array}{c}\text { Thermal } \\
\text { conductivity } \\
/(\mathrm{W} / \mathrm{mK})\end{array}$ & $\begin{array}{c}\text { Specific heat } \\
\text { capacity } \\
/(\mathrm{J} / \mathrm{kgK})\end{array}$ \\
\hline water & $10^{-9} / 2.25$ & 0.595 & 4178.5 \\
\hline Sediment & $10^{-9} / 88.378$ & 0.269 & 920 \\
\hline
\end{tabular}

Table 2. The physical parameters of sediment suspension

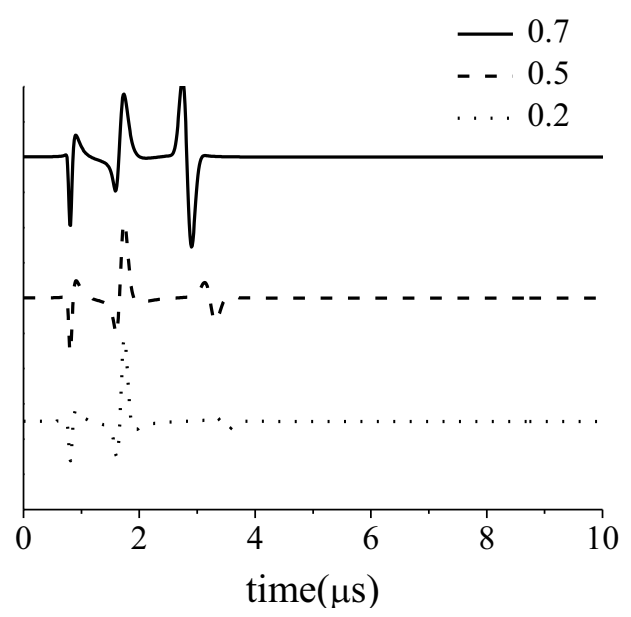

Figure 2. Interface waveforms of different sediment content at $5 \mathrm{~mm}$ of UA model

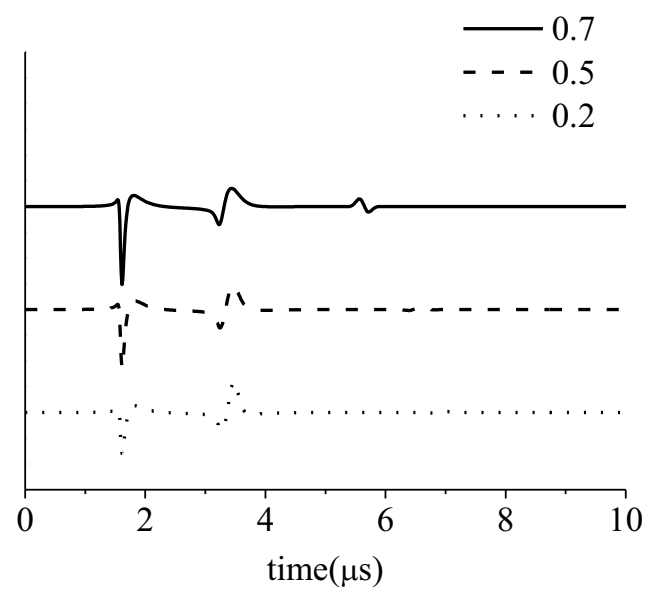

Figure 3. Interface waveforms of different sediment content at $10 \mathrm{~mm}$ of UA model 


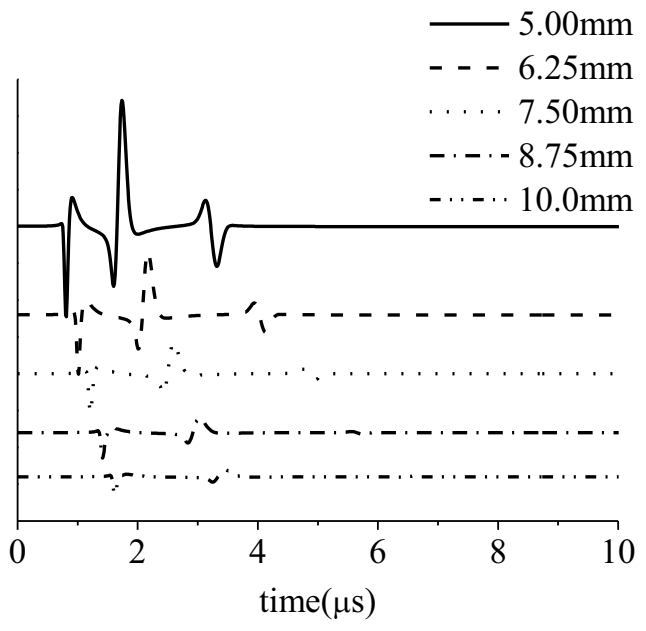

Figure4. UA model interface waveforms of different location (The sediment content is 0.5 )

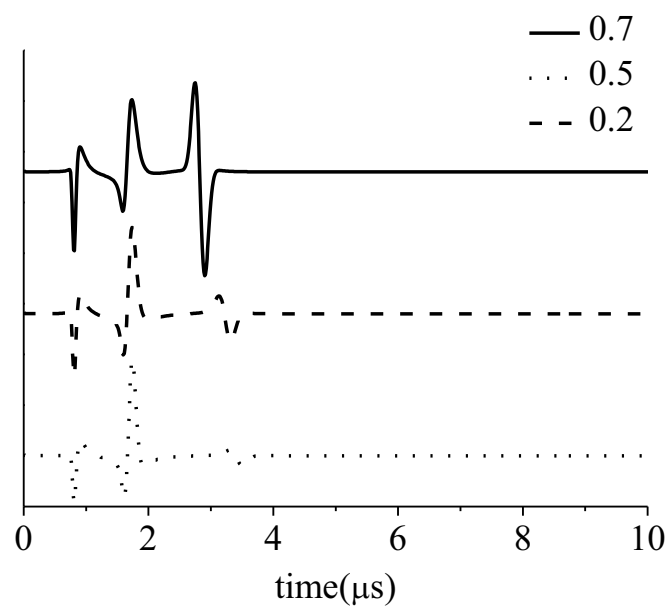

Figure 5. Interface waveforms of different sediment content at $5 \mathrm{~mm}$ of HT model

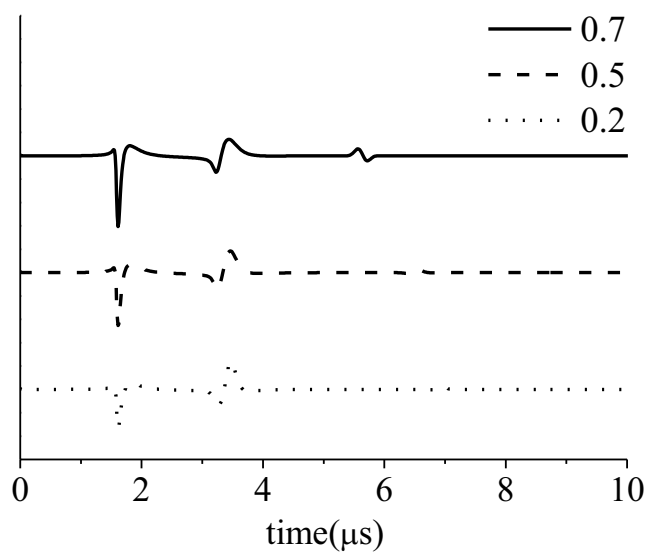

Figure6. Interface waveforms of different sediment content at $10 \mathrm{~mm}$ of HT model

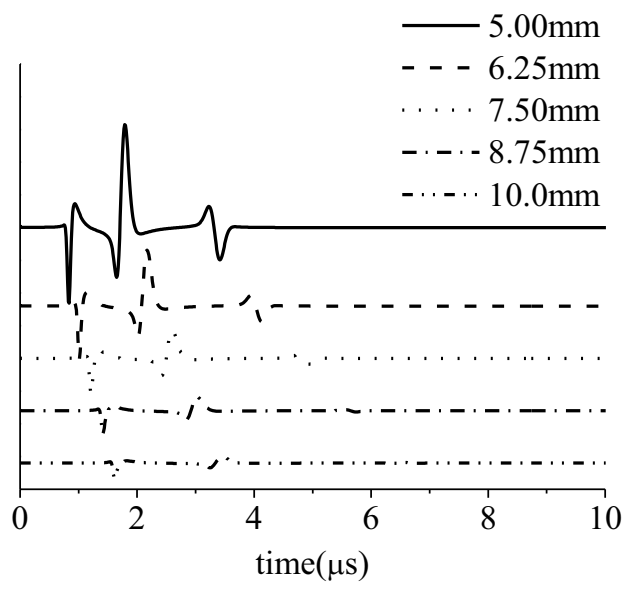

Figure7. HT model interface waveforms of different location (The sediment content is 0.5 )

From Figure 2 and Figure 3, we can obtain the interface waveforms of different sediment content at $5 \mathrm{~mm}$ and $10 \mathrm{~mm}$ of UA model. We can find that the velocity and energy of Scholte wave increases when the sediment content grows. From Fig4, we can observe the waveforms of different locations when the sediment content is 0.5. From the waveforms, we can find that the energy of each waveform declines, when the propagation distance increases.

From Figure 5 to Figure7, we can find the propagation characteristics of HT model are in good agreement with UA model.

\section{Conclusions}

In this paper, based on two models of suspension which are UA model and HT model, we get the fluid-solid interface time-domain waveform ${ }^{[7-9]}$. From the numerical results, the time-domain waveforms of UA model and HT model have a good consistency. By analyzing the influence of the sediment content, we can get the conclusion that the sediment content have a great impact on the Scholte wave and have little effect on the propagation of other waves. With the increase of the sediment content, for Scholte wave, the velocity increases and the attenuation decreases. Besides, in the same sediment content, with the distance of propagation increases, the energy of all the waves attenuate.

\section{Acknowledgements}

This work is supported by the Natural Science foundation of China Grant No.11274091,11574072 and the Fundamental Research Funds for the Central Universities of Hohai 


\section{References}

1. Mingxu Su, Xiaoshu Cai, Progress and Status ultrasonic particle detection technology and its applications in two-phase flow measurement,J. Journal of Northeastern University. 21(S1),96 99(2000).

2. Jianguo Shen, Applied Acoustics basis: the real axis integration method and two-dimensional spectroscopy (Tianjin University Press, 2004)

3. J.S. Tebbutt, R.E.Challis, Ultrasonic wave propagation in colloidal suspensions and emulsions:a comparison of four models,J. Ultrasonics.34,363-368(1996).

4. ]R.J.Urick, J.Acoust.Soc.Am.,20,283(1948).

5. Harker A H, Temple J a G, Velocity and attenuation of ultrasound in suspensions of particles in fluidsJ. J Phys D.Appl Phys, 21,1576 1588(1988)

6. Riebel U,KytoemaaH K,Theory of Sound Propagation in Suspensions:A Guide to Partical Size and Cncentration Characterization,J Powder Technol,82,115 121(1998)

7. J. G. Scholt. The range of existence of Rayleigh and Stoneley Waves[J]. Mo. Not. Roy. Astronomical Soc, 5,120-126(1947)

8. Zhu JY, Popovics JS, Analytical study of excitation and measurement of fluid-solid interface wave,J. Geophysical research letter,33(11),09 14(2006).

9. Favretto-Anres N, Rabau G. Excitation of the Stoneley-Scholte wave at the boundary between an ideal fluid and a viscoelastic solid, J. Journal of sound and vibration, 203(2), 193-208(1997) 